\title{
Moderately suppressed dimension-five proton decay in a flipped SU(5) model
}

\author{
Naoyuki Haba ${ }^{a}$ and Toshifumi Yamada ${ }^{b}$ \\ ${ }^{a}$ Institute of Science and Engineering, Shimane University, \\ Matsue 690-8504, Japan \\ ${ }^{b}$ Department of Physics, Kyoto University, Kyoto, \\ Kyoto 606-8502, Japan \\ E-mail: haba@riko.shimane-u.ac.jp, yamada.toshifumi.5f@kyoto-u.ac.jp
}

ABSTRACT: We study colored Higgsino-mediated proton decay (dimension-five proton decay) in a model based on the flipped SU(5) GUT. In the model, the GUT-breaking 10, $\overline{\mathbf{1 0}}$ fields have a GUT-scale mass term and gain VEVs through higher-dimensional operators, which induces an effective mass term between the color triplets in the $\mathbf{5}, \overline{\mathbf{5}}$ Higgs fields that is not much smaller than the GUT scale. This model structure gives rise to observable dimension-five proton decay, and at the same time achieves moderate suppression on dimension-five proton decay that softens the tension with the current bound on $\Gamma\left(p \rightarrow K^{+} \bar{\nu}\right)$. We investigate the flavor dependence of the Wilson coefficients of the operators relevant to dimension-five proton decay, by relating them with diagonalized Yukawa couplings and CKM matrix components in MSSM, utilizing the fact that the GUT Yukawa couplings are in one-to-one correspondence with the MSSM Yukawa couplings in flipped models. Then we numerically evaluate the Wilson coefficients, and predict the distributions of the ratios of the partial widths of various proton decay modes.

KeYwords: GUT, Supersymmetric Standard Model

ArXiv EPrint: 2110.01198 


\section{Contents}

1 Introduction 1

2 Model 2

3 Wilson coefficients contributing to proton decay 5

$4 \quad$ Flavor dependence of Wilson coefficients $\quad 7$

$\begin{array}{llr}5 & \text { Numerical analysis } & 9\end{array}$

$\begin{array}{llr}6 & \text { Summary } & 15\end{array}$

\section{Introduction}

Proton decay mediated by colored Higgsinos in supersymmetric grand unified theories (SUSY GUTs) [1, 2], called dimension-five proton decay, is a primary target in the proton decay searches at HyperKamiokande [3], JUNO [4], and DUNE [5, 6]. This is because the GUT gauge boson mass is predicted to be $\sim 2 \cdot 10^{16} \mathrm{GeV}$ in usual SUSY GUTs and the corresponding partial width of GUT gauge boson-mediated proton decay is out of the sensitivity ranges of the above experiments (however, proton decay mediated by $(\mathbf{3}, \mathbf{2}, 1 / 6)$ gauge boson in SUSY SO(10) GUT can be accessible [7]). In non-SUSY GUTs, GUT gauge boson-mediated proton decay can be within the experimental reach, and one can even set upper bounds on the proton lifetime in some cases [8]. Nevertheless, dependence on the choice of split SU(5) multiplets that assist the gauge coupling unification, is inevitable. In contrast, if Nature favors as light SUSY particles as possible (e.g., the SUSY particle mass spectrum is such that the $p \rightarrow K^{+} \bar{\nu}$ partial width is narrowly above the current experimental bound [9]) for the naturalness of the electroweak scale, there is a great chance that dimension-five proton decay is observed in forthcoming experiments. (For study on proton decay and the LHC bounds on SUSY particle masses and the Higgs particle mass, see refs. $[10,11]$.) In this situation, it is important to study the flavor dependence of dimensionfive proton decay in various SUSY GUT models, and compare the partial widths of different decay modes, as in ref. [12], to bridge theory and proton decay search experiments.

In this paper, we focus on dimension-five proton decay in a model based on the SUSY flipped SU(5) GUT $[13,14] .{ }^{1}$ Although the flipped SU(5) GUT by itself cannot address the origin of the $\mathrm{U}(1)$ hypercharge quantization, it has attractive features such as the realization of the doublet-triplet splitting, and the suppression of dimension-five proton decay that allows one to lower the sfermion mass without conflicting the current bound

\footnotetext{
${ }^{1} \mathrm{~A}$ non-SUSY model has first appeared in ref. [15].
} 
on the $p \rightarrow K^{+} \bar{\nu}$ decay. In pervasive models of the SUSY flipped SU(5) GUT, dimensionfive proton decay is in effect totally suppressed, because the mass term between the color triplets in the $\mathbf{5}, \overline{\mathbf{5}}$ Higgs fields and that between the color triplets in the GUT-breaking $\mathbf{1 0}, \overline{\mathbf{1 0}}$ fields, are at the soft SUSY breaking scale, not at the GUT scale. However, if the GUT-breaking 10, $\overline{\mathbf{1 0}}$ fields are allowed to possess a GUT-scale mass term and gain vacuum expectation values (VEVs) through higher-dimensional operators, then the color triplets in

the $\mathbf{5}, \overline{\mathbf{5}}$ fields gain an effective mass term not much smaller than the GUT scale, which gives rise to observable dimension-five proton decays. Meanwhile, the operators obtained after integrating out the colored Higgsinos can be $O(100)$ times suppressed compared to those in non-flipped models, which mitigates the tension with the current experimental bound on the $p \rightarrow K^{+} \bar{\nu}$ mode. We materialize the above possibility in our model of the SUSY flipped SU(5) GUT, and investigate the flavor dependence of dimension-five proton decay in the model. Interestingly, since the GUT Yukawa couplings for the $\mathbf{5}, \overline{\mathbf{5}}$ Higgs fields are in one-to-one correspondence with the Yukawa couplings of the minimal SUSY Standard Model (MSSM), we have a strong predictive power on the flavor structure of the Wilson coefficients of the operators relevant to dimension-five proton decay. We take advantage of the above feature and express the Wilson coefficients in terms of diagonalized Yukawa couplings and Cabibbo-Kobayashi-Maskawa (CKM) matrix components in MSSM, plus one unknown unitary matrix and several unknown phases. Then we vary the unknown unitary matrix and phases and predict the distributions of the partial width ratios of different proton decay modes.

Previously, colored Higgsino-mediated proton decay in the SUSY flipped SU(5) GUT has been studied in ref. [16]. However, since the mass term between the color triplets of the $\mathbf{5}, \overline{\mathbf{5}}$ Higgs fields and that between the color triplets of the GUT-breaking 10, $\overline{\mathbf{1 0}}$ fields are highly suppressed, only chirality non-flipping colored Higgsino exchange is considered, unlike the present paper where we focus on chirality flipping colored Higgsino exchange. For reference, GUT gauge boson-mediated proton decay in SUSY flipped SU(5) GUT models has been investigated in refs. [17]-[24].

This paper is organized as follows. In section 2, we describe our model of the SUSY flipped SU(5) GUT. In section 3, we present the expressions for the Wilson coefficients of dimension-five operators obtained after integrating out colored Higgs fields and dimensionsix operators obtained after integrating out the SUSY particles which contribute to proton decay. In section 4, we investigate the flavor dependence of the Wilson coefficients by relating them with diagonalized Yukawa couplings and CKM matrix in MSSM. In section 5, we numerically evaluate the Wilson coefficients using the values of diagonalized Yukawa couplings and CKM matrix based on experimental data, and randomly varying the remaining unknown parameters. The results are presented as a prediction for the distributions of proton decay partial width ratios. Section 6 summarizes the paper.

\section{Model}

We consider a model based on $\mathrm{SU}(5) \times \mathrm{U}(1)_{X}$ gauge group and the $Z_{2}$ matter parity. The field content is as follows: three generations of chiral superfields in $(\mathbf{1 0}, 1),(\overline{\mathbf{5}},-3)$, 


\begin{tabular}{|c|c|c|c|}
\hline chiral superfield & $\mathrm{SU}(5)$ & $\mathrm{U}(1)_{X}$ & $R$-parity \\
\hline $\mathbf{1 0}_{1}^{i}$ & $\mathbf{1 0}$ & 1 & -1 \\
$\overline{\mathbf{5}}_{-3}^{i}$ & $\overline{\mathbf{5}}$ & -3 & -1 \\
$\mathbf{1}_{5}^{i}$ & $\mathbf{1}$ & 5 & -1 \\
\hline$H$ & $\mathbf{1 0}$ & 1 & +1 \\
$\bar{H}$ & $\overline{\mathbf{1 0}}$ & -1 & +1 \\
$h$ & $\mathbf{5}$ & -2 & +1 \\
$\bar{h}$ & $\overline{\mathbf{5}}$ & 2 & +1 \\
\hline$\Sigma$ & $\mathbf{2 4}$ & 0 & +1 \\
\hline$S^{i}$ & $\mathbf{1}$ & 0 & -1 \\
\hline
\end{tabular}

Table 1. Field content. $i$ labels the three generations.

$(\mathbf{1}, 5)$ representations of $\mathrm{SU}(5) \times \mathrm{U}(1)_{X}$ and with $Z_{2}$ matter parity -1 , denoted by $\mathbf{1 0}_{1}^{i}$, $\overline{\mathbf{5}}_{-3}^{i}, \mathbf{1}_{5}^{i}$ where $i$ labels the three generations; chiral superfields in $(\mathbf{1 0}, 1),(\overline{\mathbf{1 0}},-1)$ representations and with $Z_{2}$ matter parity +1 , denoted by $H, \bar{H}$; chiral superfields in $(\mathbf{5},-2)$, $(\overline{\mathbf{5}}, 2)$ representations and with $Z_{2}$ matter parity +1 , denoted by $h, \bar{h}$. Additionally, we introduce a chiral superfield in $(\mathbf{2 4}, 0)$ representation with $Z_{2}$ matter parity +1 , denoted by $\Sigma$, and three generations of chiral superfields in $(\mathbf{1}, 0)$ representation with $Z_{2}$ matter parity -1 , denoted by $S^{i}$. The field content is summarized in table 1 . The fundamental superpotential reads

$$
\begin{aligned}
W^{\prime}= & \left(Y_{d}\right)_{i j} \mathbf{1 0}_{1}^{i} \mathbf{1 0}_{1}^{j} h+\left(Y_{u}\right)_{i j} \mathbf{1 0}_{1}^{i} \overline{\mathbf{5}}_{-3}^{j} \bar{h}+\left(Y_{e}\right)_{i j} \overline{\mathbf{5}}_{-3}^{i} \mathbf{1}_{5}^{j} h \\
& +\mu_{h} h \bar{h}+\lambda H H h+\lambda^{\prime} \bar{H} \bar{H} \bar{h}+M \bar{H} H \\
& +\kappa \Sigma \bar{H} H+\frac{1}{2} M_{\Sigma} \Sigma^{2}+\frac{1}{3} \lambda_{\Sigma} \Sigma^{3} \\
& +\left(Y_{S}\right)_{i j} \mathbf{1 0}_{1}^{i} S^{j} \bar{H}+\frac{1}{2} M_{S^{i}} S^{i} S^{i} .
\end{aligned}
$$

Here we assume that there are no higher-dimensional operators at this stage. The mass terms for the $\Sigma$ and $S^{i}$ fields are assumed to originate from some Planck-scale physics and their masses $M_{\Sigma}, M_{S^{i}}$ are about the reduced Planck mass $M_{P}=2.4 \cdot 10^{18} \mathrm{GeV}$. On the other hand, $\mu_{h}$ is at the soft SUSY breaking scale, while $M$ is about (GUT scale) ${ }^{2} / M_{P}$, as shown later. The origin of the hierarchy $\left|\mu_{h}\right| \ll|M|$ can be explained by introducing an $R$-symmetry under which $H, \bar{H}$ and the matter superfields have $R$-charge 1 and $h, \bar{h}$ have 0 and by assuming that the Planck-scale physics that gives rise to $M_{\Sigma}, M_{S^{i}}$ does not respect this $R$-symmetry. By integrating out $\Sigma$ and $S^{i}$, we obtain the following effective superpotential at scales below $M_{P}$ :

$$
\begin{aligned}
W= & \left(Y_{d}\right)_{i j} \mathbf{1 0}_{1}^{i} \mathbf{1 0}_{1}^{j} h+\left(Y_{u}\right)_{i j} \mathbf{1 0}_{1}^{i} \overline{\mathbf{5}}_{-3}^{j} \bar{h}+\left(Y_{e}\right)_{i j} \overline{\mathbf{5}}_{-3}^{i} \mathbf{1}_{5}^{j} h \\
& +\mu_{h} h \bar{h}+\lambda H H h+\lambda^{\prime} \bar{H} \bar{H} \bar{h}+M \bar{H} H \\
& -\frac{\kappa^{2}}{2 M_{\Sigma}} \bar{H} H \bar{H} H-\frac{\left(Y_{S}\right)_{i k}\left(Y_{S}\right)_{j k}}{2 M_{S^{k}}}\left(\bar{H} \mathbf{1 0} \mathbf{0}_{1}^{i}\right)\left(\bar{H} \mathbf{1 0} \mathbf{0}_{1}^{j}\right),
\end{aligned}
$$


where $\bar{H} H \bar{H} H$ stands for the coupling where SU(5) indices are summed cyclically and $\left(\bar{H} 10_{1}^{i}\right)\left(\bar{H} 10_{1}^{j}\right)$ the couplings where SU(5) indices are summed separately in each bracket. The $\bar{H} H \bar{H} H$ operator, obtained by integrating out $\Sigma$, triggers GUT breaking, while the $\left(\bar{H} 10_{1}^{i}\right)\left(\bar{H} 10_{1}^{j}\right)$ operators, obtained by integrating out $S^{i}$ 's, generate the Majorana mass of the singlet neutrinos. The isospin-doublet components of $h, \bar{h}$ gain mass only from the $\mu_{h} h \bar{h}$ term, while the color-triplet components additionally gain GUT-scale mass from the $\lambda H H h+\bar{\lambda} \bar{H} \bar{H} \bar{h}$ terms after GUT-breaking, which achieves the doublet-triplet splitting. Note that the effective superpotential eq. (2.2) does not contain higher-dimensional operators giving rise to proton decay $\mathbf{1 0}_{1}^{i} \mathbf{1 0}_{1}^{j} \mathbf{1 0}_{1}^{k} \overline{\mathbf{5}}_{-3}^{\ell}$; proton decay occurs only through the exchange of colored Higgsinos, colored Higgs bosons and GUT gauge bosons (the contributions of the latter two can be neglected in the present model).

Let us write the Standard Model (SM) gauge-singlet, color-triplet and color-anti-triplet components of $H$ as $N_{H}^{c}, Q_{H}, D_{H}^{c}$, and the SM gauge-singlet, color-anti-triplet and colortriplet components of $\bar{H}$ as $\bar{N}_{H}^{c}, \bar{Q}_{H}, \bar{D}_{H}^{c}$, respectively. $N_{H}^{c}, \bar{N}_{H}^{c}$ develop VEVs as

$$
\left\langle N_{H}^{c}\right\rangle\left\langle\bar{N}_{H}^{c}\right\rangle=2 M M_{\Sigma} / \kappa^{2}, \quad\left|\left\langle N_{H}^{c}\right\rangle\right|=\left|\left\langle\bar{N}_{H}^{c}\right\rangle\right|
$$

and break $\mathrm{SU}(5) \times \mathrm{U}(1)_{X}$ into the $\mathrm{SM}$ gauge group. The $Q_{H}, \bar{Q}_{H}$ fields are would-be Nambu-Goldstone modes eaten by the GUT gauge boson. Along the GUT breaking, $h$ is decomposed into isospin-doublet $H_{d}$ and color-triplet $\bar{D}_{h}^{c}$, and $\bar{h}$ into isospin-doublet $H_{u}$ and color-anti-triplet $D_{h}^{c}$. The $H_{d}, H_{u}$ fields are identified with the MSSM down-type and up-type Higgs fields, respectively. The $\bar{D}_{h}^{c}, \bar{D}_{H}^{c}, D_{h}^{c}, D_{H}^{c}$ fields constitute the colored Higgs fields. Along the GUT breaking, $\mathbf{1 0}_{1}^{i}, \overline{\mathbf{5}}_{-3}^{i}, \mathbf{1}_{5}^{i}$ fields are decomposed as

$$
\mathbf{1 0}_{1}^{i} \rightarrow Q^{i}, D^{c i}, N^{c i}, \quad \overline{\mathbf{5}}_{-3}^{i} \rightarrow U^{c i}, L^{i}, \quad \mathbf{1}_{5}^{i} \rightarrow E^{c i}
$$

where $Q^{i}, U^{c i}, D^{c i}, L^{i}, E^{c i}, N^{c i}$ are identified with the MSSM quark doublets, up-type quark singlets, down-type quark singlets, lepton doublets, charged leptons, and the singlet neutrinos, respectively. Couplings $\left(Y_{d}\right)_{i j},\left(Y_{u}\right)_{i j},\left(Y_{e}\right)_{i j}$ are identified with the down-type quark, up-type quark and charged lepton Yukawa couplings of MSSM, respectively.

The tiny active neutrino mass is generated via the Type-1 seesaw mechanism [25]-[29]. The Majorana mass term for the singlet neutrinos is generated from the second term of the third line of eq. (2.2) along the GUT breaking as

$$
W \supset-\frac{1}{2} N^{c i} N^{c j} \frac{\left(Y_{S}\right)_{i k}\left(Y_{S}\right)_{j k}}{2 M_{S^{k}}}\left\langle\bar{N}_{H}^{c}\right\rangle^{2} .
$$

Since the Dirac mass for $N^{c i}$ and $L^{j}$ is given by $\left(Y_{u}\right)_{i j} v_{u} / \sqrt{2}$ with $v_{u}=\sin \beta \cdot 246 \mathrm{GeV}$, the active neutrino mass matrix is obtained as

$$
M_{\nu}=Y_{u}^{T} Y_{S}^{T-1} M_{S} Y_{S}^{-1} Y_{u} \frac{v_{u}^{2}}{\left\langle\overline{\left.N_{H}^{c}\right\rangle^{2}}\right.}
$$

where $M_{S}=\operatorname{diag}\left(M_{S^{1}}, M_{S^{2}}, M_{S^{3}}\right)$. We estimate the scale of the components of $Y_{S}$. Consider a special case where $M_{S}=M_{P} \operatorname{diag}(1,1,1)$ and $Y_{u}$ is given by

$$
Y_{u}=\operatorname{diag}\left(y_{u}, y_{c}, y_{t}\right) U_{M N S}^{\dagger}
$$


in the basis where the lepton-doublet components of $\overline{\mathbf{5}}_{-3}^{i}$ diagonalize the charged lepton mass matrix. Here $U_{M N S}$ denotes Maki-Nakagawa-Sakata matrix [30]. In this case, we get

$$
Y_{S}=\operatorname{diag}\left(y_{u} / \sqrt{m_{1}}, y_{c} / \sqrt{m_{2}}, y_{t} / \sqrt{m_{3}}\right) \frac{v_{u} \sqrt{M_{P}}}{\left\langle\bar{N}_{H}^{c}\right\rangle},
$$

where $m_{1}, m_{2}, m_{3}$ are the active neutrino masses. For $\left|\left\langle\bar{N}_{H}^{c}\right\rangle\right| \simeq 3 \cdot 10^{16} \mathrm{GeV}, v_{u} \simeq 246 \mathrm{GeV}$, and for the normal hierarchy with $m_{2} \gg m_{1}$, the following numerical values reproduce the measured neutrino mass differences:

$$
Y_{S}=\operatorname{diag}(a, 0.006,0.9) \quad \text { with } a>10^{-4} .
$$

We expect that in general cases suppression of some components of $Y_{S}$ at $O\left(10^{-3}\right)$ suffices to reproduce the measured neutrino mass differences.

\section{Wilson coefficients contributing to proton decay}

We focus on proton decay mediated by colored Higgsinos. The mass matrix for the colored Higgs fields reads

$$
W \supset\left(\begin{array}{ll}
D_{h}^{c} & D_{H}^{c}
\end{array}\right) \mathcal{M}_{H_{C}}\left(\begin{array}{c}
\bar{D}_{h}^{c} \\
\bar{D}_{H}^{c}
\end{array}\right), \quad \mathcal{M}_{H_{C}}=\left(\begin{array}{cc}
\mu_{h} & \lambda^{\prime}\left\langle\bar{N}_{H}^{c}\right\rangle \\
\lambda\left\langle N_{H}^{c}\right\rangle & M
\end{array}\right) .
$$

The colored Higgs fields couple to the matter fields as

$$
\begin{aligned}
W \supset & \left(Y_{d}\right)_{i j} Q^{i} Q^{j} \bar{D}_{h}^{c}+\left(Y_{d}\right)_{i j} D^{c i} N^{c j} \bar{D}_{h}^{c}+\left(Y_{u}\right)_{i j} Q^{i} L^{j} D_{h}^{c} \\
& +\left(Y_{u}\right)_{i j} D^{c i} U^{c j} D_{h}^{c}+\left(Y_{e}\right)_{i j} U^{c i} E^{c j} \bar{D}_{h}^{c} .
\end{aligned}
$$

Integrating out the colored Higgs fields, we obtain the following dimension-five operators responsible for proton decay:

$$
-W_{5}=\frac{1}{2} C_{5 L}^{i j k l}\left(Q^{k} Q^{l}\right)\left(Q^{i} L^{j}\right)+C_{5 R}^{i j k l} E^{c k} U^{c l} U^{c i} D^{c j}
$$

where in the first term isospin indices are summed in each bracket, and the Wilson coefficients satisfy

$$
\begin{aligned}
& C_{5 L}^{i j k l}\left(\mu=\mu_{H_{C}}\right)=\left.\left(\mathcal{M}_{H_{C}}^{-1}\right)_{11}\left\{\left(Y_{d}\right)_{k l}\left(Y_{u}\right)_{i j}-\frac{1}{2}\left(Y_{d}\right)_{l i}\left(Y_{u}\right)_{k j}-\frac{1}{2}\left(Y_{d}\right)_{i k}\left(Y_{u}\right)_{l j}\right\}\right|_{\mu=\mu_{H_{C}}}, \\
& C_{5 R}^{i j k l}\left(\mu=\mu_{H_{C}}\right)=\left.\left(\mathcal{M}_{H_{C}}^{-1}\right)_{11}\left\{\left(Y_{e}\right)_{l k}\left(Y_{u}\right)_{j i}-\left(Y_{e}\right)_{i k}\left(Y_{u}\right)_{j l}\right\}\right|_{\mu=\mu_{H_{C}}}
\end{aligned}
$$

where $\mu$ denotes the renormalization scale and $\mu_{H_{C}}$ is about the colored Higgs mass eigenvalues. The effective inverse of the $D_{h}^{c}, \bar{D}_{h}^{c}$ fields, $\left(\mathcal{M}_{H_{C}}^{-1}\right)_{11}$, is obtained from eq. (3.1) as

$$
\left(\mathcal{M}_{H_{C}}^{-1}\right)_{11}=-\frac{M}{\lambda \lambda^{\prime}\left\langle N_{H}^{c}\right\rangle\left\langle\bar{N}_{H}^{c}\right\rangle}=-\frac{\kappa^{2}}{2 \lambda \lambda^{\prime} M_{\Sigma}},
$$


where we have used the fact that $\mu_{h}$ is negligible compared to the GUT-scale, and used eq. (2.3) in the second equality. When $\left|\kappa^{2} /\left(2 \lambda \lambda^{\prime}\right)\right|=1$ and $M_{\Sigma}=M_{P}$, the Wilson coefficients $C_{5 L}^{i j k l}, C_{5 R}^{i j k l}$ are about 100 times suppressed compared to non-flipped models, where $\left(\mathcal{M}_{H_{C}}^{-1}\right)_{11}$ is given by the inverse of the colored Higgs mass $\sim 2 \cdot 10^{16} \mathrm{GeV}$. The resulting $10^{4}$ suppression on proton decay partial widths allows the model to evade the current stringent experimental bound on the $p \rightarrow K^{+} \bar{\nu}$ decay without enormously raising sfermion masses. Still, the suppression is not strong, and leaves the possibility of observing proton decay in near-future experiments.

Integrating out the SUSY particles, we obtain the following operators responsible for proton decay:

$$
-\mathcal{L}_{6}=C_{L L}^{i j k l}\left(\psi_{u_{L}}^{k} \psi_{d_{L}}^{l}\right)\left(\psi_{d_{L}}^{i} \psi_{\nu_{L}}^{j}\right)+\bar{C}_{L L}^{i j k l}\left(\psi_{u_{L}}^{k} \psi_{d_{L}}^{l}\right)\left(\psi_{u_{L}}^{i} \psi_{e_{L}}^{j}\right)+C_{R L}^{i j k l}\left(\psi_{\nu_{L}}^{k} \psi_{d_{L}}^{l}\right)\left(\psi_{u_{R}^{c}}^{i} \psi_{d_{R}^{c}}^{j}\right)(3
$$

where $\psi$ denotes a SM Weyl spinor and spinor index is summed in each bracket. Those Wilson coefficients which contribute to proton decay are $C_{L L}^{d \alpha u d}, C_{L L}^{s \alpha u d}, C_{L L}^{d \alpha u s}, \bar{C}_{L L}^{u \beta u d}$, $\bar{C}_{L L}^{u \beta u s}, C_{R L}^{u d \tau d}, C_{R L}^{u d \tau s}, C_{R L}^{u s \tau d}$ with $\alpha=e, \mu, \tau$ and $\beta=e, \mu$. They satisfy, at the soft SUSY breaking scale $\mu=\mu_{\mathrm{SUSY}}{ }^{2}$

$$
\begin{aligned}
& C_{L L}^{d \alpha u d}\left(\mu_{\mathrm{SUSY}}\right)=\left.\frac{M_{\widetilde{W}}}{m_{\tilde{q}}^{2}} \mathcal{F}\left(\left|M_{\widetilde{W}}\right|^{2} / m_{\tilde{q}}^{2}, m_{\tilde{\ell}^{\alpha}}^{2} / m_{\tilde{q}}^{2}\right) g_{2}^{2}\left(C_{5 L}^{d \alpha u d}-C_{5 L}^{u \alpha d d}\right)\right|_{\mu=\mu_{\mathrm{SUSY}}}, \\
& C_{L L}^{s \alpha u d}\left(\mu_{\mathrm{SUSY}}\right)=\left.\frac{M_{\widetilde{W}}}{m_{\tilde{q}}^{2}} \mathcal{F}\left(\left|M_{\widetilde{W}}\right|^{2} / m_{\tilde{q}}^{2}, m_{\tilde{\ell}^{\alpha}}^{2} / m_{\tilde{q}}^{2}\right) g_{2}^{2}\left(C_{5 L}^{s \alpha u d}-C_{5 L}^{u \alpha d s}\right)\right|_{\mu=\mu_{\mathrm{SUSY}}}, \\
& C_{L L}^{d \alpha u s}\left(\mu_{\mathrm{SUSY}}\right)=\left.\frac{M_{\widetilde{W}}}{m_{\tilde{q}}^{2}} \mathcal{F}\left(\left|M_{\widetilde{W}}\right|^{2} / m_{\tilde{q}}^{2}, m_{\tilde{\ell}^{\alpha}}^{2} / m_{\tilde{q}}^{2}\right) g_{2}^{2}\left(C_{5 L}^{d \alpha u s}-C_{5 L}^{u \alpha d s}\right)\right|_{\mu=\mu_{\mathrm{SUSY}}}, \\
& \bar{C}_{L L}^{u \beta u d}\left(\mu_{\mathrm{SUSY}}\right)=\left.\frac{M_{\widetilde{W}}}{m_{\tilde{q}}^{2}} \mathcal{F}\left(\left|M_{\widetilde{W}}\right|^{2} / m_{\tilde{q}}^{2}, m_{\tilde{\ell}^{\beta}}^{2} / m_{\tilde{q}}^{2}\right) g_{2}^{2}\left(-C_{5 L}^{u \beta u d}+C_{5 L}^{d \beta u u}\right)\right|_{\mu=\mu_{\mathrm{SUSY}}}, \\
& \bar{C}_{L L}^{u \beta u s}\left(\mu_{\mathrm{SUSY}}\right)=\left.\frac{M_{\widetilde{W}}}{m_{\tilde{q}}^{2}} \mathcal{F}\left(\left|M_{\widetilde{W}}\right|^{2} / m_{\tilde{q}}^{2}, m_{\tilde{\ell}^{\beta}}^{2} / m_{\tilde{q}}^{2}\right) g_{2}^{2}\left(-C_{5 L}^{u \beta u s}+C_{5 L}^{s \beta u u}\right)\right|_{\mu=\mu_{\mathrm{SUSY}}}, \\
& C_{R L}^{u d \tau d}\left(\mu_{\mathrm{SUSY}}\right)=\left.\frac{\mu_{h}}{m_{\tilde{t}_{R}}^{2}} \tilde{\mathcal{F}}\left(\left|\mu_{h}\right|^{2} / m_{\tilde{t}_{R}}^{2}, m_{\tilde{\tau}_{R}}^{2} / m_{\tilde{t}_{R}}^{2}\right)\left(V_{t d}^{\text {ckm }}\right)^{*} y_{t} y_{\tau} C_{5 R}^{u d \tau t}\right|_{\mu=\mu_{\mathrm{SUSY}}}, \\
& C_{R L}^{u d \tau s}\left(\mu_{\mathrm{SUSY}}\right)=\left.\frac{\mu_{h}}{m_{\tilde{t}_{R}}^{2}} \tilde{\mathcal{F}}\left(\left|\mu_{h}\right|^{2} / m_{\tilde{t}_{R}}^{2}, m_{\tilde{\tau}_{R}}^{2} / m_{\tilde{t}_{R}}^{2}\right)\left(V_{t s}^{\mathrm{ckm}}\right)^{*} y_{t} y_{\tau} C_{5 R}^{u d \tau t}\right|_{\mu=\mu_{\mathrm{SUSY}}} \\
& C_{R L}^{u s \tau d}\left(\mu_{\mathrm{SUSY}}\right)=\left.\frac{\mu_{h}}{m_{\tilde{t}_{R}}^{2}} \tilde{\mathcal{F}}\left(\left|\mu_{h}\right|^{2} / m_{\tilde{t}_{R}}^{2}, m_{\tilde{\tau}_{R}}^{2} / m_{\tilde{t}_{R}}^{2}\right)\left(V_{t d}^{\mathrm{ckm}}\right)^{*} y_{t} y_{\tau} C_{5 R}^{u s \tau t}\right|_{\mu=\mu_{\mathrm{SUSY}}}
\end{aligned}
$$

Here $\mathcal{F}, \tilde{\mathcal{F}}$ are loop functions given by $\mathcal{F}(x, y)=\frac{1}{x-y}\left(\frac{x}{1-x} \log x-\frac{y}{1-y} \log y\right) / 16 \pi^{2}+$ $\frac{1}{x-1}\left(\frac{x}{1-x} \log x+1\right) / 16 \pi^{2}$ and $\tilde{\mathcal{F}}(x, y)=\frac{1}{x-y}\left(\frac{x}{1-x} \log x-\frac{y}{1-y} \log y\right) / 16 \pi^{2}$, and $M_{\widetilde{W}}, \mu_{h}, m_{\tilde{q}}$, $m_{\tilde{\ell}^{\alpha}}, m_{\tilde{t}_{R}}, m_{\tilde{\tau}_{R}}$ respectively denote the Wino mass, $\mu$-term, mass of 1 st and 2 nd generation

\footnotetext{
${ }^{2}$ When writing $C_{5 L}^{s \alpha u d}$, we mean that $Q_{i}$ is in the flavor basis where the down-type quark Yukawa coupling is diagonal and that the down-type quark component of $Q_{i}$ is exactly $s$ quark (the up-type quark component of $Q_{i}$ is a mixture of $\left.u, c, t\right)$. Likewise, $Q_{k}$ is in the flavor basis where the up-type quark Yukawa coupling is diagonal and its up-type component is exactly $u$ quark, and $Q_{l}$ is in the flavor basis where the down-type quark Yukawa coupling is diagonal and its down-type quark component is exactly $d$ quark. The same rule applies to $C_{5 L}^{u \alpha d s}$ and others.
} 
isospin-doublet squarks (assumed degenerate), mass of isospin-doublet slepton of flavor $\alpha$, mass of isospin-singlet top squark, and mass of isospin-singlet tau slepton (mixings between isospin-doublet and singlet sfermions are neglected). $y_{t}, y_{\tau}, V^{\mathrm{ckm}}$ denote the top quark and tau lepton Yukawa couplings and CKM matrix, respectively.

Finally, $C_{L L}^{d \alpha u d}, C_{L L}^{s \alpha u d}, C_{L L}^{d \alpha u s}, \bar{C}_{L L}^{u \beta u d}, \bar{C}_{L L}^{u \beta u s}, C_{R L}^{u d \tau d}, C_{R L}^{u d \tau s}, C_{R L}^{u s \tau d}$ at a hadronic scale $\mu=\mu_{\text {had }}$ determine proton decay amplitudes.

\section{Flavor dependence of Wilson coefficients}

We investigate the flavor dependence of the Wilson coefficients $C_{L L}^{d \alpha} u d, C_{L L}^{s \alpha u d}, C_{L L}^{d \alpha u s}$, $\bar{C}_{L L}^{u \beta u d}, \bar{C}_{L L}^{u \beta u s}, C_{R L}^{u d \tau d}, C_{R L}^{u d \tau s}, C_{R L}^{u s \tau d}(\alpha=e, \mu, \tau$ and $\beta=e, \mu)$ by relating them with diagonalized Yukawa couplings and the CKM matrix in MSSM. We can write

$$
\begin{aligned}
& C_{L L}^{d \alpha u d}\left(\mu_{\mathrm{had}}\right)=\left.C_{L L \alpha}^{0}\left\{\left(Y_{d}\right)_{u_{L} d_{L}}\left(Y_{u}\right)_{d_{L} \alpha_{L}}-\left(Y_{d}\right)_{d_{L} d_{L}}\left(Y_{u}\right)_{u_{L} \alpha_{L}}\right\}\right|_{\mu=\mu_{H_{C}}}, \\
& C_{L L}^{s \alpha u d}\left(\mu_{\mathrm{had}}\right)=\left.C_{L L \alpha}^{0}\left\{\left(Y_{d}\right)_{u_{L} d_{L}}\left(Y_{u}\right)_{s_{L} \alpha_{L}}-\left(Y_{d}\right)_{d_{L} s_{L}}\left(Y_{u}\right)_{u_{L} \alpha_{L}}\right\}\right|_{\mu=\mu_{H_{C}}}, \\
& C_{L L}^{d \alpha u s}\left(\mu_{\mathrm{had}}\right)=\left.C_{L L \alpha}^{0}\left\{\left(Y_{d}\right)_{u_{L} s_{L}}\left(Y_{u}\right)_{d_{L} \alpha_{L}}-\left(Y_{d}\right)_{d_{L} s_{L}}\left(Y_{u}\right)_{u_{L} \alpha_{L}}\right\}\right|_{\mu=\mu_{H_{C}}}, \\
& \bar{C}_{L L}^{u \beta u d}\left(\mu_{\mathrm{had}}\right)=\left.C_{L L \beta}^{0}\left\{\left(Y_{d}\right)_{u_{L} u_{L}}\left(Y_{u}\right)_{d_{L} \beta_{L}}-\left(Y_{d}\right)_{u_{L} d_{L}}\left(Y_{u}\right)_{u_{L} \beta_{L}}\right\}\right|_{\mu=\mu_{H_{C}}}, \\
& \bar{C}_{L L}^{u \beta u s}\left(\mu_{\mathrm{had}}\right)=\left.C_{L L \beta}^{0}\left\{\left(Y_{d}\right)_{u_{L} u_{L}}\left(Y_{u}\right)_{s_{L} \beta_{L}}-\left(Y_{d}\right)_{u_{L} s_{L}}\left(Y_{u}\right)_{u_{L} \beta_{L}}\right\}\right|_{\mu=\mu_{H_{C}}}, \\
& C_{R L}^{u d \tau d}\left(\mu_{\mathrm{had}}\right)=\left.\left.C_{R L}^{0}\left(V_{t d}^{\mathrm{ckm}}\right)^{*}\right|_{\mu=\mu_{\mathrm{SUSY}}}\left\{\left(Y_{e}\right)_{t_{R} \tau_{R}}\left(Y_{u}\right)_{d_{R} u_{R}}-\left(Y_{e}\right)_{u_{R} \tau_{R}}\left(Y_{u}\right)_{d_{R} t_{R}}\right\}\right|_{\mu=\mu_{H_{C}}}, \\
& C_{R L}^{u d \tau s}\left(\mu_{\mathrm{had}}\right)=\left.\left.C_{R L}^{0}\left(V_{t s}^{\mathrm{ckm}}\right)^{*}\right|_{\mu=\mu_{\mathrm{SUSY}}}\left\{\left(Y_{e}\right)_{t_{R} \tau_{R}}\left(Y_{u}\right)_{d_{R} u_{R}}-\left(Y_{e}\right)_{u_{R} \tau_{R}}\left(Y_{u}\right)_{d_{R} t_{R}}\right\}\right|_{\mu=\mu_{H_{C}}}, \\
& C_{R L}^{u s \tau d}\left(\mu_{\mathrm{had}}\right)=\left.\left.C_{R L}^{0}\left(V_{t d}^{\mathrm{ckm}}\right)^{*}\right|_{\mu=\mu_{\mathrm{SUSY}}}\left\{\left(Y_{e}\right)_{t_{R} \tau_{R}}\left(Y_{u}\right)_{s_{R} u_{R}}-\left(Y_{e}\right)_{u_{R} \tau_{R}}\left(Y_{u}\right)_{s_{R} t_{R}}\right\}\right|_{\mu=\mu_{H_{C}}} .
\end{aligned}
$$

Here $C_{L L \alpha}^{0}(\alpha=e, \mu, \tau), C_{R L}^{0}$ are defined as

$$
\begin{aligned}
C_{L L \alpha}^{0} & =\left.\frac{3}{2}\left(\mathcal{M}_{H_{C}}^{-1}\right)_{11} A_{L \alpha}^{\mathrm{MSSM}} \frac{M_{\widetilde{W}}}{m_{\tilde{q}}^{2}} \mathcal{F}\left(\left|M_{\widetilde{W}}\right|^{2} / m_{\tilde{q}}^{2}, m_{\tilde{\ell}^{\alpha}}^{2} / m_{\tilde{q}}^{2}\right) A_{L L}^{\mathrm{SM}} g_{2}^{2}\right|_{\mu=\mu_{\mathrm{SUSY}}}, \\
C_{R L}^{0} & =\left.\left(\mathcal{M}_{H_{C}}^{-1}\right)_{11} A_{R t \tau}^{\mathrm{MSSM}} \frac{\mu_{h}}{m_{\tilde{t}_{R}}^{2}} \tilde{\mathcal{F}}\left(\left|\mu_{h}\right|^{2} / m_{\tilde{t}_{R}}^{2}, m_{\tilde{\tau}_{R}}^{2} / m_{\tilde{t}_{R}}^{2}\right) A_{R L}^{\mathrm{SM}} y_{t} y_{\tau}\right|_{\mu=\mu_{\mathrm{SUSY}}}
\end{aligned}
$$

where $A_{L \alpha}^{\mathrm{MSSM}}$ represents renormalization group (RG) corrections for $C_{5 L}^{i j k l}$ in MSSM that involve three light-flavor (1st and 2nd generations) quarks and one lepton of flavor $\alpha$, and $A_{R t \tau}^{\mathrm{MSSM}}$ those for $C_{5 R}^{i j k l}$ in MSSM that involve two light-flavor quarks, one top quark and one tau lepton. $A_{L L}^{\mathrm{SM}}$ and $A_{R L}^{\mathrm{SM}}$ respectively represent $\mathrm{RG}$ corrections for $C_{L L}^{i j k l}, \bar{C}_{L L}^{i j k l}$ and $C_{R L}^{i j k l}$ in SM involving no top quark. When defining $A_{L \alpha}^{\mathrm{MSSM}}, A_{R t \tau}^{\mathrm{MSSM}}, A_{L L}^{\mathrm{SM}}, A_{R L}^{\mathrm{SM}}$, we neglect the 1st and 2nd generation Yukawa couplings in MSSM, and the Yukawa couplings other than the top quark's in SM. (Then we have $A_{L e}^{\mathrm{MSSM}}=A_{L \mu}^{\mathrm{MSSM}}$, but we adhere to the redundant notation with $A_{L \alpha}^{\mathrm{MSSM}}$ for brevity.)

The Yukawa coupling components in eqs. (4.1)-(4.8) are related to diagonalized Yukawa couplings and CKM matrix components as follows: since $Y_{d}$ is a symmetric matrix, and since $Y_{d}$ by definition satisfies $\left(Y_{d}\right)_{d_{L} d_{R}}=y_{d},\left(Y_{d}\right)_{s_{L} s_{R}}=y_{s},\left(Y_{d}\right)_{b_{L} b_{R}}=y_{b}$, 
$\left(Y_{d}\right)_{d_{L} s_{R}}=\left(Y_{d}\right)_{d_{L} b_{R}}=\left(Y_{d}\right)_{s_{L} d_{R}}=\left(Y_{d}\right)_{s_{L} b_{R}}=\left(Y_{d}\right)_{b_{L} d_{R}}=\left(Y_{d}\right)_{b_{L} s_{R}}=0$, we have

$$
\begin{aligned}
& \left(Y_{d}\right)_{d_{L} d_{L}}=y_{d} e^{i \phi_{1}}, \quad\left(Y_{d}\right)_{s_{L} s_{L}}=y_{s} e^{i \phi_{2}}, \quad\left(Y_{d}\right)_{b_{L} b_{L}}=y_{b} e^{i \phi_{3}}, \\
& \left(Y_{d}\right)_{d_{L} s_{L}}=\left(Y_{d}\right)_{s_{L} b_{L}}=\left(Y_{d}\right)_{b_{L} d_{L}}=\left(Y_{d}\right)_{s_{L} d_{L}}=\left(Y_{d}\right)_{b_{L} s_{L}}=\left(Y_{d}\right)_{d_{L} b_{L}}=0
\end{aligned}
$$

where $y_{d}, y_{s}, y_{b}$ denote the diagonalized Yukawa couplings for the down, strange and bottom quarks, respectively, and $\phi_{1}, \phi_{2}, \phi_{3}$ are unknown phases. Combining eq. (4.11) with the definition of the CKM matrix, we get

$$
\begin{aligned}
\left(Y_{d}\right)_{u_{L} d_{L}}= & \left(V_{u d}^{\mathrm{ckm}}\right)^{*}\left(Y_{d}\right)_{d_{L} d_{L}}=\left(V_{u d}^{\mathrm{ckm}}\right)^{*} y_{d} e^{i \phi_{1}} \\
\left(Y_{d}\right)_{u_{L} s_{L}}= & \left(V_{u s}^{\mathrm{ckm}}\right)^{*}\left(Y_{d}\right)_{s_{L} s_{L}}=\left(V_{u s}^{\mathrm{ckm}}\right)^{*} y_{s} e^{i \phi_{2}} \\
\left(Y_{d}\right)_{u_{L} u_{L}}= & \left(V_{u d}^{\mathrm{ckm}}\right)^{*}\left(Y_{d}\right)_{d_{L} d_{L}}\left(V_{u d}^{\mathrm{ckm}}\right)^{*}+\left(V_{u s}^{\mathrm{ckm}}\right)^{*}\left(Y_{d}\right)_{s_{L} s_{L}}\left(V_{u s}^{\mathrm{ckm}}\right)^{*} \\
& +\left(V_{u b}^{\mathrm{ckm}}\right)^{*}\left(Y_{d}\right)_{b_{L} b_{L}}\left(V_{u b}^{\mathrm{ckm}}\right)^{*} \\
= & \left(V_{u d}^{\mathrm{ckm}}\right)^{* 2} y_{d} e^{i \phi_{1}}+\left(V_{u s}^{\mathrm{ckm}}\right)^{* 2} y_{s} e^{i \phi_{2}}+\left(V_{u b}^{\mathrm{ckm}}\right)^{* 2} y_{b} e^{i \phi_{3}}
\end{aligned}
$$

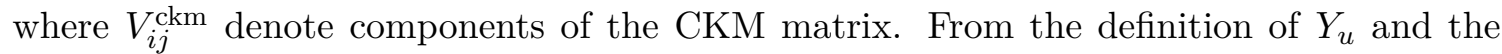
CKM matrix, we get, for $\alpha=e, \mu, \tau$,

$$
\begin{aligned}
\left(Y_{u}\right)_{u_{L} \alpha_{L}} & =\left(Y_{u}\right)_{u_{L} u_{R}} U_{u_{R} \alpha_{L}}=y_{u} U_{u_{R} \alpha_{L}} \\
\left(Y_{u}\right)_{d_{L} \alpha_{L}} & =V_{u d}^{\mathrm{ckm}}\left(Y_{u}\right)_{u_{L} u_{R}} U_{u_{R} \alpha_{L}}+V_{c d}^{\mathrm{ckm}}\left(Y_{u}\right)_{c_{L} c_{R}} U_{c_{R} \alpha_{L}}+V_{t d}^{\mathrm{ckm}}\left(Y_{u}\right)_{t_{L} t_{R}} U_{t_{R} \alpha_{L}} \\
& =V_{u d}^{\mathrm{ckm}} y_{u} U_{u_{R} \alpha_{L}}+V_{c d}^{\mathrm{ckm}} y_{c} U_{c_{R} \alpha_{L}}+V_{t d}^{\mathrm{ckm}} y_{t} U_{t_{R} \alpha_{L}}, \\
\left(Y_{u}\right)_{s_{L} \alpha_{L}} & =V_{u s}^{\mathrm{ckm}} y_{u} U_{u_{R} \alpha_{L}}+V_{c s}^{\mathrm{ckm}} y_{c} U_{c_{R} \alpha_{L}}+V_{t s}^{\mathrm{ckm}} y_{t} U_{t_{R} \alpha_{L}}
\end{aligned}
$$

where $y_{u}, y_{c}, y_{t}$ denote the diagonalized Yukawa couplings for the up, charm and top quarks, respectively, and $U_{i j}$ is a component of an unknown unitary matrix $U$ that transforms the flavor basis of $\overline{\mathbf{5}}_{-3}^{i}$ 's as

$$
\left(\begin{array}{c}
\overline{\mathbf{5}}_{-3}^{u_{R}} \\
\overline{\mathbf{5}}_{-3}^{c_{R}} \\
\overline{\mathbf{5}}_{-3}^{t_{R}}
\end{array}\right)=U\left(\begin{array}{c}
\overline{\mathbf{5}}_{-3}^{e_{L}} \\
\overline{\mathbf{5}}_{-3}^{\mu_{L}} \\
\overline{\mathbf{5}}_{-3}^{\tau_{L}}
\end{array}\right)
$$

Combining the definition of $Y_{e}$ with eq. (4.14), we get

$$
\left(Y_{e}\right)_{t_{R} \tau_{R}}=U_{t_{R} \tau_{L}}^{*} y_{\tau}, \quad\left(Y_{e}\right)_{u_{R} \tau_{R}}=U_{u_{R} \tau_{L}}^{*} y_{\tau} .
$$

Eq. (4.11) gives that the flavor basis of $\mathbf{1 0}_{1}^{i}$ is transformed as

$$
\left(\begin{array}{c}
\mathbf{1 0}_{1}^{d_{R}} \\
\mathbf{1 0}_{1}^{s_{R}} \\
\mathbf{1 0}_{1}^{b_{R}}
\end{array}\right)=\left(\begin{array}{ccc}
e^{i \phi_{1}} & 0 & 0 \\
0 & e^{i \phi_{2}} & 0 \\
0 & 0 & e^{i \phi_{3}}
\end{array}\right)\left(\begin{array}{c}
\mathbf{1 0}_{1}^{d_{L}} \\
\mathbf{1 0}_{1}^{s_{L}} \\
\mathbf{1 0}_{1}^{b_{L}}
\end{array}\right) .
$$

Combining eq. (4.16) with the definition of CKM matrix, we get

$$
\begin{array}{ll}
\left(Y_{u}\right)_{d_{R} u_{R}}=e^{-i \phi_{1}}\left(Y_{u}\right)_{d_{L} u_{R}}=e^{-i \phi_{1}} V_{u d}^{\mathrm{ckm}} y_{u}, \quad\left(Y_{u}\right)_{d_{R} t_{R}}=e^{-i \phi_{1}}\left(Y_{u}\right)_{d_{L} t_{R}}=e^{-i \phi_{1}} V_{t d}^{\mathrm{ckm}} y_{t}, \\
\left(Y_{u}\right)_{s_{R} u_{R}}=e^{-i \phi_{2}}\left(Y_{u}\right)_{s_{L} u_{R}}=e^{-i \phi_{2}} V_{u s}^{\mathrm{ckm}} y_{u}, \quad\left(Y_{u}\right)_{s_{R} t_{R}}=e^{-i \phi_{2}}\left(Y_{u}\right)_{s_{L} t_{R}}=e^{-i \phi_{2}} V_{t s}^{\mathrm{ckm}} y_{t} .
\end{array}
$$


Inserting eqs. (4.11)-(4.13), (4.15), (4.17) into eqs. (4.1)-(4.8), we obtain

$$
\begin{aligned}
& C_{L L}^{d \alpha u d}\left(\mu_{\mathrm{had}}\right) / C_{L L \alpha}^{0}=e^{i \phi_{1}}\left\{\left(V_{u d}^{\mathrm{ckm}}\right)^{*} y_{d}\left(V_{u d}^{\mathrm{ckm}} y_{u} U_{u_{R} \alpha_{L}}+V_{c d}^{\mathrm{ckm}} y_{c} U_{c_{R} \alpha_{L}}+V_{t d}^{\mathrm{ckm}} y_{t} U_{t_{R} \alpha_{L}}\right)\right. \\
& \left.-y_{d} y_{u} U_{u_{R} \alpha_{L}}\right\} \\
& C_{L L}^{s \alpha} u d\left(\mu_{\mathrm{had}}\right) / C_{L L \alpha}^{0}=e^{i \phi_{1}}\left(V_{u d}^{\mathrm{ckm}}\right)^{*} y_{d}\left(V_{u s}^{\mathrm{ckm}} y_{u} U_{u_{R} \alpha_{L}}+V_{c s}^{\mathrm{ckm}} y_{c} U_{c_{R} \alpha_{L}}+V_{t s}^{\mathrm{ckm}} y_{t} U_{t_{R} \alpha_{L}}\right) \\
& C_{L L}^{d \alpha u s}\left(\mu_{\mathrm{had}}\right) / C_{L L \alpha}^{0}=e^{i \phi_{2}}\left(V_{u s}^{\mathrm{ckm}}\right)^{*} y_{s}\left(V_{u d}^{\mathrm{ckm}} y_{u} U_{u_{R} \alpha_{L}}+V_{c d}^{\mathrm{ckm}} y_{c} U_{c_{R} \alpha_{L}}+V_{t d}^{\mathrm{ckm}} y_{t} U_{t_{R} \alpha_{L}}\right) \\
& \bar{C}_{L L}^{u \beta u d}\left(\mu_{\mathrm{had}}\right) / C_{L L \beta}^{0}=\left\{\left(\left(V_{u d}^{\mathrm{ckm}}\right)^{* 2} y_{d} e^{i \phi_{1}}+\left(V_{u s}^{\mathrm{ckm}}\right)^{* 2} y_{s} e^{i \phi_{2}}+\left(V_{u b}^{\mathrm{ckm}}\right)^{* 2} y_{b} e^{i \phi_{3}}\right)\right. \\
& \times\left(V_{u d}^{\mathrm{ckm}} y_{u} U_{u_{R} \beta_{L}}+V_{c d}^{\mathrm{ckm}} y_{c} U_{c_{R} \beta_{L}}+V_{t d}^{\mathrm{ckm}} y_{t} U_{t_{R} \beta_{L}}\right) \\
& \left.-\left(V_{u d}^{\mathrm{ckm}}\right)^{*} y_{d} e^{i \phi_{1}} y_{u} U_{u_{R} \beta_{L}}\right\} \text {, } \\
& \bar{C}_{L L}^{u \beta u s}\left(\mu_{\mathrm{had}}\right) / C_{L L \beta}^{0}=\left\{\left(\left(V_{u d}^{\mathrm{ckm}}\right)^{* 2} y_{d} e^{i \phi_{1}}+\left(V_{u s}^{\mathrm{ckm}}\right)^{* 2} y_{s} e^{i \phi_{2}}+\left(V_{u b}^{\mathrm{ckm}}\right)^{* 2} y_{b} e^{i \phi_{3}}\right)\right. \\
& \times\left(V_{u s}^{\mathrm{ckm}} y_{u} U_{u_{R} \beta_{L}}+V_{c s}^{\mathrm{ckm}} y_{c} U_{c_{R} \beta_{L}}+V_{t s}^{\mathrm{ckm}} y_{t} U_{t_{R} \beta_{L}}\right) \\
& \left.-\left(V_{u s}^{\mathrm{ckm}}\right)^{*} y_{s} e^{i \phi_{2}} y_{u} U_{u_{R} \beta_{L}}\right\} \text {, } \\
& C_{R L}^{u d \tau d}\left(\mu_{\mathrm{had}}\right) / C_{R L}^{0}=e^{-i \phi_{1}}\left(\bar{V}_{t d}^{\mathrm{ckm}}\right)^{*} y_{\tau}\left(U_{t_{R} \tau_{L}}^{*} V_{u d}^{\mathrm{ckm}} y_{u}-U_{u_{R} \tau_{L}}^{*} V_{t d}^{\mathrm{ckm}} y_{t}\right), \\
& C_{R L}^{u d \tau s}\left(\mu_{\mathrm{had}}\right) / C_{R L}^{0}=e^{-i \phi_{1}}\left(\bar{V}_{t s}^{\mathrm{ckm}}\right)^{*} y_{\tau}\left(U_{t_{R} \tau_{L}}^{*} V_{u d}^{\mathrm{ckm}} y_{u}-U_{u_{R} \tau_{L}}^{*} V_{t d}^{\mathrm{ckm}} y_{t}\right), \\
& C_{R L}^{u s \tau d}\left(\mu_{\mathrm{had}}\right) / C_{R L}^{0}=e^{-i \phi_{2}}\left(\bar{V}_{t d}^{\mathrm{ckm}}\right)^{*} y_{\tau}\left(U_{t_{R} \tau_{L}}^{*} V_{u s}^{\mathrm{ckm}} y_{u}-U_{u_{R} \tau_{L}}^{*} V_{t s}^{\mathrm{ckm}} y_{t}\right),
\end{aligned}
$$

where the diagonalized Yukawa couplings and CKM matrix components are evaluated at scale $\mu=\mu_{H_{C}}$, except for $\bar{V}_{t s}^{\mathrm{ckm}}, \bar{V}_{t d}^{\mathrm{ckm}}$, which are evaluated at $\mu=\mu_{\mathrm{SUSY}}$. The right-hand sides of eqs. (4.18)-(4.25) contain diagonalized Yukawa couplings and components of the CKM matrix, and mostly determine the order of magnitude of each Wilson coefficient.

\section{Numerical analysis}

We numerically evaluate the right-hand sides of eqs. (4.18)-(4.25) by randomly varying the unknown unitary matrix $U$ and unknown phases $\phi_{1}, \phi_{2}, \phi_{3}$. The result is presented in the form of the ratios of the proton decay partial widths below,

$$
\begin{aligned}
& \Gamma\left(p \rightarrow \pi^{+} \bar{\nu}\right)=\sum_{\alpha=e, \mu, \tau} \Gamma\left(p \rightarrow \pi^{+} \bar{\nu}_{\alpha}\right), \\
& \Gamma\left(p \rightarrow K^{+} \bar{\nu}\right)=\sum_{\alpha=e, \mu, \tau} \Gamma\left(p \rightarrow K^{+} \bar{\nu}_{\alpha}\right), \\
& \Gamma\left(p \rightarrow \pi^{0} \beta^{+}\right), \\
& \Gamma\left(p \rightarrow \eta \beta^{+}\right), \\
& \Gamma\left(p \rightarrow K^{0} \beta^{+}\right),
\end{aligned}
$$

which are suitable for the presentation because they are observable quantities. 
The partial width of each mode is given by

$$
\begin{aligned}
\Gamma\left(p \rightarrow \pi^{+} \bar{\nu}_{\beta}\right)= & \mathcal{C}\left|\beta_{H}\left(\mu_{\mathrm{had}}\right) \frac{1}{f_{\pi}}(1+D+F) C_{L L}^{d \beta u d}\left(\mu_{\mathrm{had}}\right)\right|^{2} \\
& (\beta=e, \mu) \\
\Gamma\left(p \rightarrow \pi^{+} \bar{\nu}_{\tau}\right)= & \mathcal{C}\left|\beta_{H}\left(\mu_{\mathrm{had}}\right) \frac{1}{f_{\pi}}(1+D+F) C_{L L}^{d \tau u d}\left(\mu_{\mathrm{had}}\right)+\alpha_{H}\left(\mu_{\mathrm{had}}\right) \frac{1}{f_{\pi}} C_{R L}^{u d \tau d}\left(\mu_{\mathrm{had}}\right)\right|^{2} \\
\Gamma\left(p \rightarrow K^{+} \bar{\nu}_{\beta}\right)= & \mathcal{C}\left|\beta_{H}\left(\mu_{\mathrm{had}}\right) \frac{1}{f_{\pi}}\left\{\left(1+\frac{D}{3}+F\right) C_{L L}^{s \beta u d}\left(\mu_{\mathrm{had}}\right)+\frac{2 D}{3} C_{L L}^{d \beta u s}\left(\mu_{\mathrm{had}}\right)\right\}\right|^{2} \\
& (\beta=e, \mu), \\
\Gamma\left(p \rightarrow K^{+} \bar{\nu}_{\tau}\right)= & \mathcal{C} \mid \beta_{H}\left(\mu_{\mathrm{had}}\right) \frac{1}{f_{\pi}}\left\{\left(1+\frac{D}{3}+F\right) C_{L L}^{s \tau} u d\left(\mu_{\mathrm{had}}\right)+\frac{2 D}{3} C_{L L}^{d \tau u s}\left(\mu_{\mathrm{had}}\right)\right\} \\
\Gamma\left(p \rightarrow \pi^{0} \beta^{+}\right)= & \mathcal{C}\left|\beta_{H}\left(\mu_{\mathrm{had}}\right) \frac{1}{f_{\pi}} \frac{1}{\sqrt{2}}\left\{\left(1+\frac{D}{3}+F\right) C_{R L}^{u d \tau s}\left(\mu_{\mathrm{had}}\right)+\frac{2 D}{3} C_{R L}^{u s \tau}\left(\mu_{\mathrm{had}}\right)\right\}\right|^{2}, \\
\Gamma\left(p \rightarrow \eta \beta^{+}\right)= & \left.\mathcal{C}\left|\beta_{H}\left(\mu_{\mathrm{had}}\right) \frac{1}{f_{\pi}} \sqrt{\frac{3}{2}}\left(1-\frac{D}{3}+F\right) \bar{C}_{L L}^{u \beta u d}\left(\mu_{\mathrm{had}}\right)\right|^{2 \beta u d}\left(\mu_{\mathrm{had}}\right)\right|^{2} \\
\Gamma\left(p \rightarrow K^{0} \beta^{+}\right)= & \mathcal{C}\left|\beta_{H}\left(\mu_{\mathrm{had}}\right) \frac{1}{f_{\pi}}(1-D+F) \bar{C}_{L L}^{u \beta u s}\left(\mu_{\mathrm{had}}\right)\right|^{2} \\
& (\beta=e, \mu),
\end{aligned}
$$

where $\mathcal{C}=\frac{m_{N}}{64 \pi}\left(1-\frac{m_{K}^{2}}{m_{N}^{2}}\right)^{2}$, and $\alpha_{H}, \beta_{H}$ denote hadronic matrix elements, and $D, F$ are parameters of the baryon chiral Lagrangian. The mass splittings among nucleons and hyperons are neglected.

In the evaluation of the proton decay partial widths, the baryon chiral Lagrangian parameters are given by $D=0.804, F=0.463$, and the hadronic matrix elements are taken from ref. [31] as $\alpha_{H}\left(\mu_{\mathrm{had}}\right)=-\beta_{H}\left(\mu_{\mathrm{had}}\right)=-0.0144 \mathrm{GeV}^{3}$ for $\mu_{\mathrm{had}}=2 \mathrm{GeV}$.

In the calculation of $C_{L L \alpha}^{0}, C_{R L}^{0}$, defined in eqs. (4.9), (4.10), we assume two benchmark SUSY particle mass spectra. In one spectrum, the pole masses and $\tan \beta$ satisfy

$$
\begin{aligned}
m_{\text {sfermion }} & =m_{H^{0}}=m_{H^{ \pm}}=m_{A}=\left|M_{\widetilde{g}}\right|=\left|M_{\widetilde{W}}\right|=\left|\mu_{h}\right|=100 \mathrm{TeV}, \\
\tan \beta & =5,
\end{aligned}
$$

and in the other spectrum, they satisfy

$$
\begin{aligned}
m_{\text {sfermion }} & =m_{H^{0}}=m_{H^{ \pm}}=m_{A}=\left|M_{\widetilde{g}}\right|=\left|M_{\widetilde{W}}\right|=\left|\mu_{h}\right|=30 \mathrm{TeV} \\
\tan \beta & =50
\end{aligned}
$$

where all the sfermions are mass-degenerate. The relative phase between $M_{\widetilde{W}}$ and $\mu_{h}$, which determines the relative phase between $C_{L L \alpha}^{0}$ and $C_{R L}^{0}$, is varied randomly. The 
first spectrum represents the case with low $\tan \beta$ and the second spectrum the case with high $\tan \beta$. In both spectra, the correct electroweak symmetry breaking and the values of $m_{H^{0}}, m_{H^{ \pm}}, m_{A}$ can be achieved by a fine-tuning of soft SUSY breaking parameters $m_{H_{u}}^{2}, m_{H_{d}}^{2}, B \mu$ (since $m_{H^{0}}, m_{H^{ \pm}}, m_{A}$ are much above the electroweak scale, the equality $m_{H^{0}}=m_{H^{ \pm}}=m_{A}$ holds to a good precision). Both spectra satisfy the 1-loop matching condition [47] for the SM Higgs quartic coupling around scale $\mu=m_{\text {sfermion }}$ (with vanishing $A$-terms) and thus can realize the correct Higgs particle mass $125 \mathrm{GeV}$. For the first spectrum, the model evades the current experimental bounds on proton decay, including the $90 \%$ CL bound on the $p \rightarrow K^{+} \bar{\nu}$ mode, $1 / \Gamma\left(p \rightarrow K^{+} \bar{\nu}\right)>5.9 \times 10^{33}$ yrs [9], when $\left(\mathcal{M}_{H_{C}}^{-1}\right)_{11} \lesssim 0.02 / M_{P}$ (slightly smaller than the estimate after eq. (3.5)). For the second spectrum, the model evades the current experimental bounds when $\left(\mathcal{M}_{H_{C}}^{-1}\right)_{11} \lesssim 2 / M_{P}$. The SUSY particle masses can be made smaller and be at TeV scale if we set $\left(\mathcal{M}_{H_{C}}^{-1}\right)_{11} \ll 1 / M_{P}$ by taking $|\kappa| \ll 1$. However, since such small $\kappa$ is not natural, we adhere to the relation $\left(\mathcal{M}_{H_{C}}^{-1}\right)_{11} \sim 1 / M_{P}$ and assume $O(10)$ to $100 \mathrm{TeV}$ SUSY particle mass spectra. We comment on the unification of the $\mathrm{SU}(3)_{C}$ and $\mathrm{SU}(2)_{L}$ gauge couplings $g_{3}, g_{2}$ under the above SUSY particle mass spectrum. Let us focus on the case with $\left|\lambda \lambda^{\prime}\right| \sim 1$ and where the GUT-breaking VEVs are smaller than the Planck scale. We note that in this case the colored Higgs masses $M_{H_{C 1}}, M_{H_{C 2}}$ originate mostly from the GUT-breaking VEVs and satisfy $M_{H_{C 1}} M_{H_{C 2}}=\left|\lambda \lambda^{\prime}\left\langle N_{H}^{c}\right\rangle\left\langle\bar{N}_{H}^{c}\right\rangle\right|{ }^{3}$ On the other hand, the GUT gauge boson mass $M_{G}$ satisfies $M_{G}^{2}=g_{23}^{2}\left|\left\langle N_{H}^{c}\right\rangle\left\langle\bar{N}_{H}^{c}\right\rangle\right|$ ( $g_{23}$ denotes the unified gauge coupling). Then we get $M_{H_{C 1}} M_{H_{C 2}} \sim M_{G}^{2}$, and we can use the 1-loop unification condition,

$$
\frac{1}{g_{3}^{2}(\mu)}-\frac{1}{g_{2}^{2}(\mu)}=2 \log \frac{\mu^{2}}{M_{H_{C 1}} M_{H_{C 2}}}+4 \log \frac{\mu}{M_{G}} .
$$

Numerically evaluating the condition, we obtain

$$
M_{G}^{2} M_{H_{C 1}} M_{H_{C 2}}=\left(1 \cdot 10^{16} \mathrm{GeV}\right)^{4}
$$

with negligible dependence on $\tan \beta$. The unification of $g_{3}, g_{2}$ constrains the colored Higgs masses and GUT gauge boson mass as above.

The RG corrections for the Wilson coefficients in MSSM and SM are calculated by using 1-loop RG equations in refs. $[32,33]$. We fix $\mu_{H_{C}}=2 \cdot 10^{16} \mathrm{GeV}$ and $\mu_{\text {SUSY }}=m_{\text {sfermion }}$.

The right-hand sides of eqs. (4.18)-(4.25) are evaluated by solving the 2-loop RG equations of the Yukawa couplings in SM and MSSM with the above SUSY particle mass spectrum. The input values of the RG equations are given in terms of quark and lepton masses and Wolfenstein parameters, and are taken from the central values of the following experimental data: the isospin-averaged quark mass and strange quark mass in $\overline{\mathrm{MS}}$ scheme are obtained from lattice calculations in refs. [34-39] as $\frac{1}{2}\left(m_{u}+m_{d}\right)(2 \mathrm{GeV})=3.373(80) \mathrm{MeV}$ and $m_{s}(2 \mathrm{GeV})=92.0(2.1) \mathrm{MeV}$. The up and down quark mass ratio is obtained from an estimate in ref. [40] as $m_{u} / m_{d}=0.46(3)$. The $\overline{\mathrm{MS}}$ charm and bottom quark masses are obtained from QCD sum rule calculations in ref. [41] as $m_{c}(3 \mathrm{GeV})=0.986-9\left(\alpha_{s}^{(5)}\left(M_{Z}\right)-0.1189\right) / 0.002 \pm 0.010 \mathrm{GeV}$ and

\footnotetext{
${ }^{3}$ To see this, note that the vacuum condition eq. (2.3) gives $\left|\left\langle N_{H}^{c}\right\rangle\right|=\left|\left\langle\bar{N}_{H}^{c}\right\rangle\right| \gg M$. Then the mass matrix eq. (3.1) leads to $M_{H_{C 1}} M_{H_{C 2}}=\left|\lambda \lambda^{\prime}\left\langle N_{H}^{c}\right\rangle\left\langle\bar{N}_{H}^{c}\right\rangle\right|$.
} 
$m_{b}\left(m_{b}\right)=4.163+7\left(\alpha_{s}^{(5)}\left(M_{Z}\right)-0.1189\right) / 0.002 \pm 0.014 \mathrm{GeV}$. The top quark pole mass is obtained from the latest measurement of the CMS Collaboration [43] as $170.5 \pm 0.8 \mathrm{GeV}$. The values of the Wolfenstein parameters are taken from the CKM fitter result [42]. For the QCD and QED gauge couplings, we use $\alpha_{s}^{(5)}\left(M_{Z}\right)=0.1181$ and $\alpha^{(5)}\left(M_{Z}\right)=1 / 127.95$. For the lepton and W, Z, Higgs pole masses, we use the values in Particle Data Group [44].

We comment on the impact of the choice of the benchmark spectrum in eq. (5.13). If the spectrum deviates from eq. (5.13) and the masses of isospin-doublet sleptons $\tilde{\ell}^{\alpha}$ are split, this gives rise to a splitting in $C_{L L \alpha}^{0}$ 's, which affects proton decay partial width ratios. However, since the right-hand sides of eqs. (4.18)-(4.25) have a large hierarchy, the possible splitting in $C_{L L \alpha}^{0}$ 's has only a minor impact on proton decay partial width ratios. Likewise, a splitting in the 1st and 2nd generation isospin-doublet squark masses does not change the result significantly.

The unknown unitary matrix $U$ is varied with the Haar measure given by $[45,46]$

$$
\begin{aligned}
U= & e^{i \eta} e^{i \omega_{1} \lambda_{3}+i \omega_{2} \lambda_{8}}\left(\begin{array}{ccc}
1 & 0 & 0 \\
0 & \cos \theta_{23} & \sin \theta_{23} \\
0 & -\sin \theta_{23} & \cos \theta_{23}
\end{array}\right)\left(\begin{array}{ccc}
\cos \theta_{13} & 0 & \sin \theta_{23} e^{-i \delta} \\
0 & 1 & 0 \\
-\sin \theta_{23} e^{i \delta} & 0 & \cos \theta_{13}
\end{array}\right) \\
& \times\left(\begin{array}{ccc}
\cos \theta_{12} & \sin \theta_{12} & 0 \\
-\sin \theta_{12} & \cos \theta_{12} & 0 \\
0 & 0 & 1
\end{array}\right) e^{i \chi_{1} \lambda_{3}+i \chi_{2} \lambda_{8}}, \\
\mathrm{~d} U= & \mathrm{d} \sin ^{2} \theta_{23} \mathrm{~d}_{\cos ^{4} \theta_{13}} \mathrm{~d} \sin ^{2} \theta_{12} \mathrm{~d} \eta \mathrm{d} \omega_{1} \mathrm{~d} \omega_{2} \mathrm{~d} \delta \mathrm{d} \chi_{1} \mathrm{~d} \chi_{2},
\end{aligned}
$$

where $\lambda_{3}=\operatorname{diag}(1,-1,0), \lambda_{8}=\operatorname{diag}(1,1,-2) / \sqrt{3}$. The use of the Haar measure is justifiable because we have no information on the flavor basis of $\overline{\mathbf{5}}_{-3}^{i}$ 's and the Haar measure is invariant under an arbitrary unitary transformation on the basis. The unknown phases $\phi_{1}, \phi_{2}, \phi_{3}$ and the relative phase between $M_{\widetilde{W}}$ and $\mu_{h}$ are varied with the flat distribution.

We present the result of the numerical analysis. Since $\Gamma\left(p \rightarrow K^{+} \bar{\nu}\right)$ is the largest partial width in the entire parameter space, the phenomenologically most meaningful quantities are the ratios of $\Gamma\left(p \rightarrow K^{+} \bar{\nu}\right)$ and the other partial widths. Therefore, we show the distributions of

$$
\frac{\Gamma\left(p \rightarrow \pi^{+} \bar{\nu}\right)}{\Gamma\left(p \rightarrow K^{+} \bar{\nu}\right)}, \quad \frac{\Gamma\left(p \rightarrow \pi^{0} \beta^{+}\right)}{\Gamma\left(p \rightarrow K^{+} \bar{\nu}\right)}, \quad \frac{\Gamma\left(p \rightarrow \eta \beta^{+}\right)}{\Gamma\left(p \rightarrow K^{+} \bar{\nu}\right)}, \quad \frac{\Gamma\left(p \rightarrow K^{0} \beta^{+}\right)}{\Gamma\left(p \rightarrow K^{+} \bar{\nu}\right)} \quad(\beta=e, \mu)
$$

corresponding to randomly varied values of the unknown unitary matrix $U$, unknown phases $\phi_{1}, \phi_{2}, \phi_{3}$, and relative phase between $M_{\widetilde{W}}$ and $\mu_{h}$. Figures 1 and 2 are the distributions for $\tan \beta=5,50$, respectively.

From figures 1, 2, we find that for low $\tan \beta$ such as $\tan \beta=5$, the ratio $\frac{\Gamma\left(p \rightarrow \pi^{+} \bar{\nu}\right)}{\Gamma\left(p \rightarrow K^{+} \bar{\nu}\right)}$ can be in the range 0.1-0.2 with $O(0.1)$ probability. Therefore, future sensitivity study on the $p \rightarrow \pi^{+} \bar{\nu}$ mode should be performed seriously, to examine the possibility of observing both decay modes. The other partial width ratios are mostly below 0.05 for both low and high $\tan \beta$. However, for low $\tan \beta$ such as $\tan \beta=5$, there is a non-negligible probability that $\frac{\Gamma\left(p \rightarrow \pi^{0} \beta^{+}\right)}{\Gamma\left(p \rightarrow K^{+} \bar{\nu}\right)}$ and $\frac{\Gamma\left(p \rightarrow \eta \beta^{+}\right)}{\Gamma\left(p \rightarrow K^{+} \bar{\nu}\right)}$ are in the range 0.05-0.1. In this case, the current bound on $\Gamma\left(p \rightarrow K^{+} \bar{\nu}\right)[9]$ and the future sensitivity reach for $\Gamma\left(p \rightarrow \pi^{0} \beta^{+}\right)[3]$ imply that $p \rightarrow \pi^{0} \beta^{+}$, 

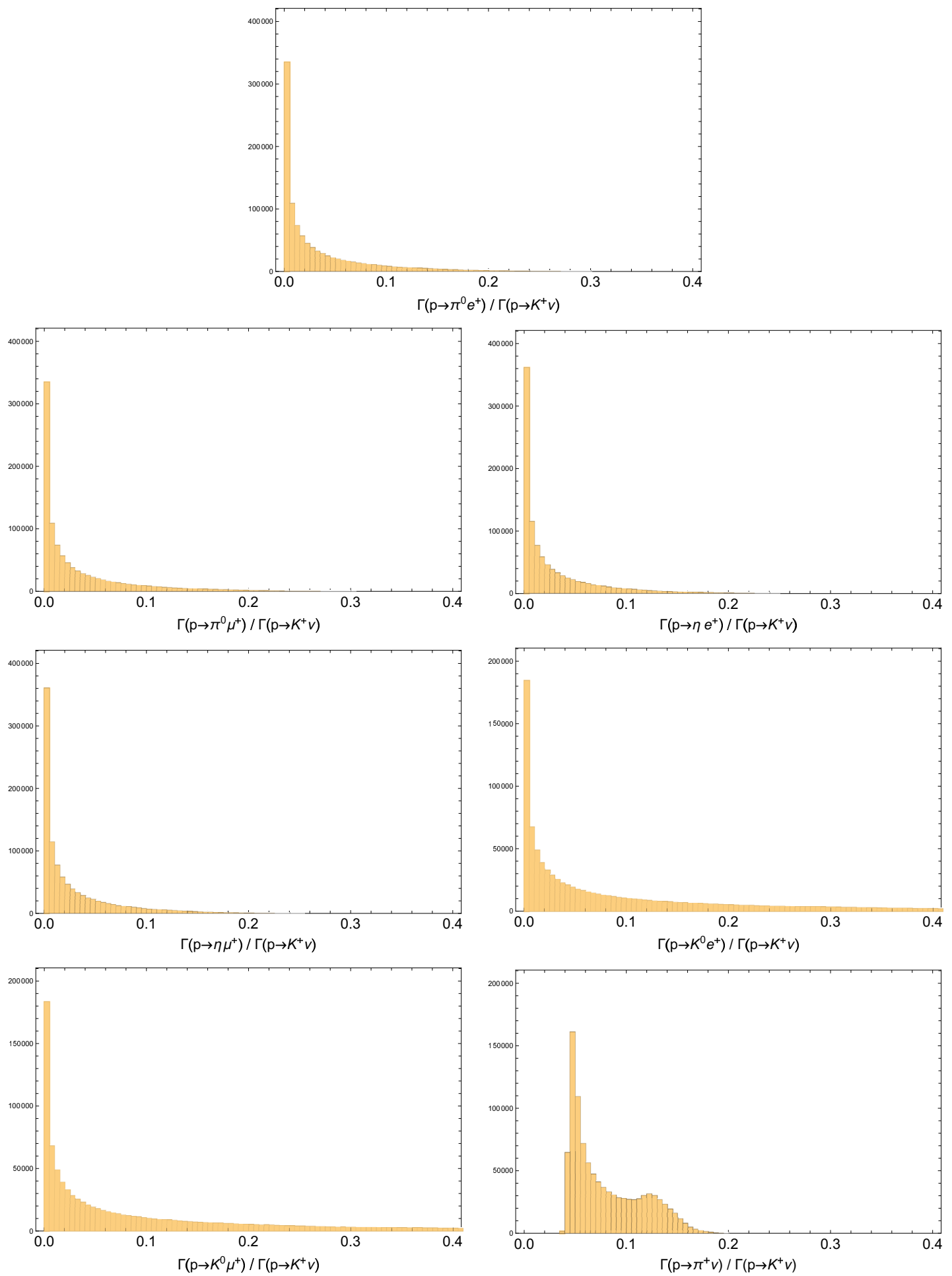

Figure 1. Distributions of proton decay partial width ratios corresponding to randomly varied values of the unknown unitary matrix $U$, unknown phases $\phi_{1}, \phi_{2}, \phi_{3}$, and relative phase between $M_{\widetilde{W}}$ and $\mu_{h}$. The vertical axis is linear and in arbitrary units. Here we assume $\tan \beta=5$. 

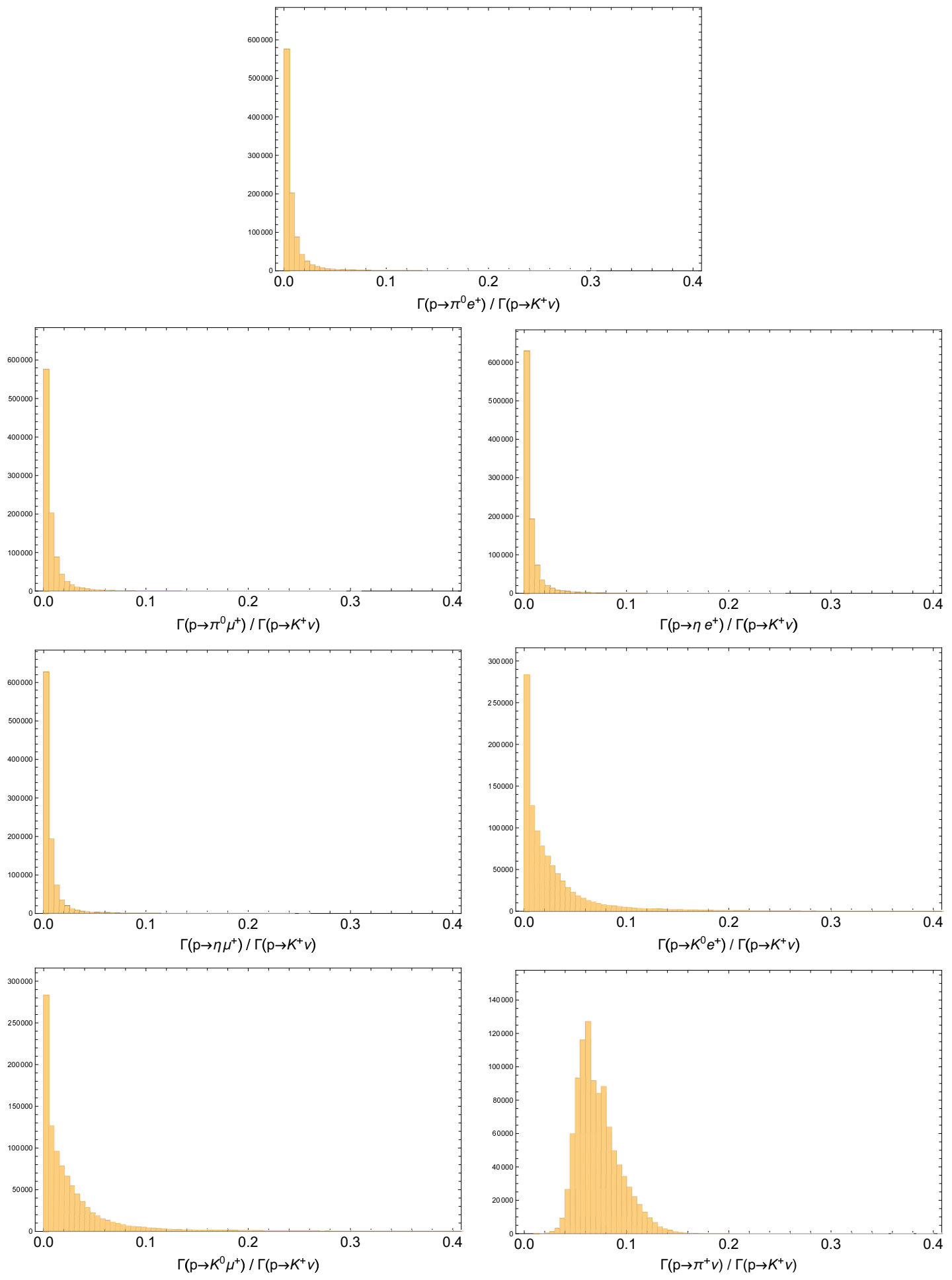

Figure 2. Same as figure 1 except that we assume $\tan \beta=50$. 
along with $p \rightarrow K^{+} \bar{\nu}$, can be discovered. Also, the distributions of $\frac{\Gamma\left(p \rightarrow K^{0} \beta^{+}\right)}{\Gamma\left(p \rightarrow K^{+} \bar{\nu}\right)}$ show a long tail above 0.05 , which indicates that there is a non-zero probability that $p \rightarrow K^{0} \beta^{+}$can be discovered with a large rate with $\frac{\Gamma\left(p \rightarrow K^{0} \beta^{+}\right)}{\Gamma\left(p \rightarrow K^{+} \bar{\nu}\right)}=O(0.1)$.

\section{Summary}

We have studied dimension-five proton decay in a model based on the flipped SU(5) GUT. In the model, the GUT-breaking $\mathbf{1 0}, \overline{\mathbf{1 0}}$ fields have a GUT-scale mass term and gain VEVs through operators suppressed by the Planck scale. This structure induces an effective mass term not much smaller than the GUT scale between the color triplets in the $\mathbf{5}, \overline{\mathbf{5}}$ Higgs fields. This mass term gives rise to observable dimension-five proton decay, and at the same time achieves moderate suppression on dimension-five proton decay amplitudes, which is estimated to be 0.01 if the coefficients in the superpotential eq. (2.2) satisfies $\left|\gamma_{1} /\left(\lambda \lambda^{\prime}\right)\right|=1$.

We have investigated the flavor structure of the Wilson coefficients of the operators contributing to dimension-five proton decay, and expressed them in terms of diagonalized Yukawa couplings and CKM matrix components in MSSM plus an unknown unitary matrix $U$ and unknown phases. We have numerically evaluated the Wilson coefficients by randomly varying $U$ and the unknown phases, and presented the result in the form of the distributions of the partial width ratios of various proton decay modes for a benchmark SUSY particle spectrum. We have found that the ratio $\frac{\Gamma\left(p \rightarrow \pi^{+} \bar{\nu}\right)}{\Gamma\left(p \rightarrow K^{+} \bar{\nu}\right)}$ can be in the range 0.1-0.2 with $O(0.1)$ probability for low $\tan \beta$ such as $\tan \beta=5$. Also, for such low $\tan \beta$, it is possible that $\frac{\Gamma\left(p \rightarrow \pi^{0} \beta^{+}\right)}{\Gamma\left(p \rightarrow K^{+} \bar{\nu}\right)}$ and $\frac{\Gamma\left(p \rightarrow \eta \beta^{+}\right)}{\Gamma\left(p \rightarrow K^{+} \bar{\nu}\right)}$ are in the range 0.05-0.1, and there is a non-zero probability that $\frac{\Gamma\left(p \rightarrow K^{0} \beta^{+}\right)}{\Gamma\left(p \rightarrow K^{+} \bar{\nu}\right)}=O(0.1)$.

\section{Acknowledgments}

This work is partially supported by Scientific Grants by the Ministry of Education, Culture, Sports, Science and Technology of Japan Nos. 17K05415 and 21H000761 (N.H.) and No. 19K147101 (T.Y.).

Open Access. This article is distributed under the terms of the Creative Commons Attribution License (CC-BY 4.0), which permits any use, distribution and reproduction in any medium, provided the original author(s) and source are credited.

\section{References}

[1] S. Weinberg, Supersymmetry at ordinary energies. 1. Masses and conservation laws, Phys. Rev. D 26 (1982) 287 [inSPIRE].

[2] N. Sakai and T. Yanagida, Proton decay in a class of supersymmetric grand unified models, Nucl. Phys. B 197 (1982) 533 [inSPIRE].

[3] Hyper-Kamiokande collaboration, Hyper-Kamiokande design report, arXiv: 1805.04163 [INSPIRE].

[4] JUNO collaboration, Neutrino physics with JUNO, J. Phys. G 43 (2016) 030401 [arXiv: 1507.05613] [INSPIRE]. 
[5] DUNE collaboration, Long-Baseline Neutrino Facility (LBNF) and Deep Underground Neutrino Experiment (DUNE): conceptual design report, volume 2: the physics program for DUNE at LBNF, arXiv:1512.06148 [INSPIRE].

[6] DUNE collaboration, Deep Underground Neutrino Experiment (DUNE), Far Detector Technical Design Report, volume II: DUNE physics, arXiv:2002.03005 [INSPIRE].

[7] N. Haba, Y. Mimura and T. Yamada, Detectable dimension-6 proton decay in SUSY SO(10) GUT at Hyper-Kamiokande, JHEP 07 (2019) 155 [arXiv: 1904.11697] [INSPIRE].

[8] N. Haba, Y. Mimura and T. Yamada, Proton lifetime upper bound in non-SUSY SU(5) GUT, Phys. Rev. D 99 (2019) 075018 [arXiv:1812.08521] [INSPIRE].

[9] Super-Kamiokande collaboration, Search for proton decay via $p \rightarrow \nu K^{+}$using 260 kiloton. year data of Super-Kamiokande, Phys. Rev. D 90 (2014) 072005 [arXiv:1408.1195] [INSPIRE].

[10] J. Ellis, J.L. Evans, N. Nagata, K.A. Olive and L. Velasco-Sevilla, Supersymmetric proton decay revisited, Eur. Phys. J. C 80 (2020) 332 [arXiv: 1912.04888] [INSPIRE].

[11] K.S. Babu, I. Gogoladze and C.S. Un, Proton lifetime in minimal SUSY SU(5) in light of LHC results, arXiv:2012.14411 [INSPIRE].

[12] N. Haba, Y. Mimura and T. Yamada, Enhanced $\Gamma\left(p \rightarrow K^{0} \mu^{+}\right) / \Gamma\left(p \rightarrow K^{+} \bar{\nu}_{\mu}\right)$ as a signature of minimal renormalizable SUSY SO(10) GUT, PTEP 2020 (2020) 093B01 [arXiv: 2002.11413] [INSPIRE].

[13] J.P. Derendinger, J.E. Kim and D.V. Nanopoulos, Anti-SU(5), Phys. Lett. B 139 (1984) 170.

[14] I. Antoniadis, J.R. Ellis, J.S. Hagelin and D.V. Nanopoulos, Supersymmetric flipped SU(5) revitalized, Phys. Lett. B 194 (1987) 231 [InSPIRE].

[15] S.M. Barr, A new symmetry breaking pattern for $\mathrm{SO}(10)$ and proton decay, Phys. Lett. B 112 (1982) 219 [INSPIRE].

[16] M. Mehmood, M.U. Rehman and Q. Shafi, Observable proton decay in flipped $\mathrm{SU}(5)$, JHEP 02 (2021) 181 [arXiv:2010.01665] [INSPIRE].

[17] J.R. Ellis, J.L. Lopez, D.V. Nanopoulos and K.A. Olive, Flipped angles and phases: a systematic study, Phys. Lett. B 308 (1993) 70 [hep-ph/9303307] [INSPIRE].

[18] J.R. Ellis, J.L. Lopez and D.V. Nanopoulos, Lowering $\alpha_{s}$ by flipping SU(5), Phys. Lett. B 371 (1996) 65 [hep-ph/9510246] [INSPIRE].

[19] J.R. Ellis, D.V. Nanopoulos and J. Walker, Flipping SU(5) out of trouble, Phys. Lett. B $\mathbf{5 5 0}$ (2002) 99 [hep-ph/0205336] [INSPIRE].

[20] I. Dorsner and P. Fileviez Perez, Distinguishing between SU(5) and flipped SU(5), Phys. Lett. B 605 (2005) 391 [hep-ph/0409095] [INSPIRE].

[21] T. Li, D.V. Nanopoulos and J.W. Walker, Fast proton decay, Phys. Lett. B 693 (2010) 580 [arXiv: 0910.0860] [INSPIRE].

[22] T. Li, D.V. Nanopoulos and J.W. Walker, Elements of F-ast proton decay, Nucl. Phys. B 846 (2011) 43 [arXiv: 1003.2570] [INSPIRE].

[23] J. Ellis, M.A.G. Garcia, N. Nagata, D.V. Nanopoulos and K.A. Olive, Proton decay: flipped vs. unflipped $\mathrm{SU}(5)$, JHEP 05 (2020) 021 [arXiv: 2003.03285] [INSPIRE]. 
[24] K. Hamaguchi, S. Hor and N. Nagata, R-symmetric flipped SU(5), JHEP 11 (2020) 140 [arXiv:2008.08940] [INSPIRE].

[25] P. Minkowski, $\mu \rightarrow$ er at a rate of one out of $10^{9}$ muon decays?, Phys. Lett. B 67 (1977) 421 [INSPIRE].

[26] T. Yanagida, Horizontal gauge symmetry and masses of neutrinos, Conf. Proc. C $\mathbf{7 9 0 2 1 3 1}$ (1979) 95 [inSPIRE].

[27] M. Gell-Mann, P. Ramond and R. Slansky, Complex spinors and unified theories, Conf. Proc. C 790927 (1979) 315 [arXiv: 1306.4669] [INSPIRE].

[28] S.L. Glashow, The future of elementary particle physics, NATO Sci. Ser. B 61 (1980) 687 [INSPIRE].

[29] R.N. Mohapatra and G. Senjanović, Neutrino mass and spontaneous parity nonconservation, Phys. Rev. Lett. 44 (1980) 912 [inSPIRE].

[30] Z. Maki, M. Nakagawa and S. Sakata, Remarks on the unified model of elementary particles, Prog. Theor. Phys. 28 (1962) 870 [INSPIRE].

[31] Y. Aoki, T. Izubuchi, E. Shintani and A. Soni, Improved lattice computation of proton decay matrix elements, Phys. Rev. D 96 (2017) 014506 [arXiv:1705.01338] [INSPIRE].

[32] J. Hisano, H. Murayama and T. Yanagida, Nucleon decay in the minimal supersymmetric SU(5) grand unification, Nucl. Phys. B 402 (1993) 46 [hep-ph/9207279] [INSPIRE].

[33] T. Goto and T. Nihei, Effect of RRRR dimension five operator on the proton decay in the minimal SU(5) SUGRA GUT model, Phys. Rev. D 59 (1999) 115009 [hep-ph/9808255] [INSPIRE].

[34] MILC collaboration, MILC results for light pseudoscalars, PoS(CD09) 007 [arXiv:0910.2966] [INSPIRE].

[35] S. Dürr et al., Lattice QCD at the physical point: light quark masses, Phys. Lett. B 701 (2011) 265 [arXiv: 1011.2403] [INSPIRE].

[36] S. Dürr et al., Lattice QCD at the physical point: simulation and analysis details, JHEP 08 (2011) 148 [arXiv: 1011.2711] [InSPIRE].

[37] C. McNeile, C.T.H. Davies, E. Follana, K. Hornbostel and G.P. Lepage, High-Precision $c$ and $b$ masses, and $Q C D$ coupling from current-current correlators in lattice and continuum $Q C D$, Phys. Rev. D 82 (2010) 034512 [arXiv: 1004.4285] [InSPIRE].

[38] RBC, UKQCD collaboration, Domain wall QCD with physical quark masses, Phys. Rev. D 93 (2016) 074505 [arXiv: 1411.7017] [INSPIRE].

[39] A. Bazavov et al., Staggered chiral perturbation theory in the two-flavor case and SU(2) analysis of the MILC data, PoS(LATTICE2010) 083 [arXiv:1011.1792] [INSPIRE].

[40] S. Aoki et al., Review of lattice results concerning low-energy particle physics, Eur. Phys. J. C 77 (2017) 112 [arXiv: 1607.00299] [InSPIRE].

[41] K.G. Chetyrkin et al., Charm and bottom quark masses: an update, Phys. Rev. D 80 (2009) 074010 [arXiv: 0907.2110] [INSPIRE].

[42] CKMfitter Group collaboration, CP violation and the CKM matrix: assessing the impact of the asymmetric B factories, Eur. Phys. J. C 41 (2005) 1 [hep-ph/0406184] [InSPIRE]. 


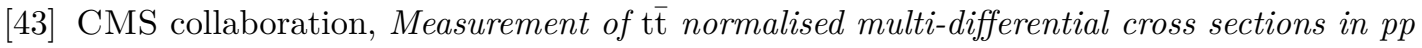
collisions at $\sqrt{s}=13 \mathrm{TeV}$, and simultaneous determination of the strong coupling strength, top quark pole mass, and parton distribution functions, Eur. Phys. J. C 80 (2020) 658 [arXiv: 1904.05237] [INSPIRE].

[44] Particle Data Group collaboration, Review of particle physics, PTEP 2020083 (2020) C01.

[45] N. Haba and H. Murayama, Anarchy and hierarchy, Phys. Rev. D 63 (2001) 053010 [hep-ph/0009174] [INSPIRE].

[46] X. Lu and H. Murayama, Neutrino mass anarchy and the universe, JHEP 08 (2014) 101 [arXiv: 1405.0547] [INSPIRE].

[47] S.A.R. Ellis and J.D. Wells, High-scale supersymmetry, the Higgs boson mass, and gauge unification, Phys. Rev. D 96 (2017) 055024 [arXiv:1706.00013] [INSPIRE]. 